\title{
LA BASE DE CONOCIMIENTO $Y$ SU IMPACTO EN LA CAPACIDAD DE ABSORCIÓN DE PYMES DE BAJA TECNOLOGÍA*
}

\author{
Jaider Vega-Jurado** \\ José Luís Polo-Otero \\ María Alejandra Cotes-Torres ${ }^{* * * * *}$

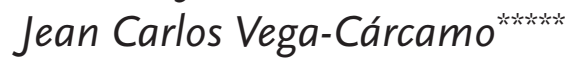

\footnotetext{
* doi: 10.11144/Javeriana.cao30-55.bcica. Este artículo es el resultado de la “investigación Implementación de un programa de gestión de la innovación empresarial para fortalecer las pymes de sectores estratégicos, Atlántico Caribe" con financiamiento del Departamento del Atlántico, Fondo de Ciencia y Tecnología, Sistema General de Regalías. El artículo se recibió el 23/01/2017 y se aprobó el 25/11/2017. Sugerencia de citación: Vega-Jurado, J., Polo-Otero, J. L., Cotes-Torres, M. A. y Vega-Cárcamo, J. C. (2017). La base de conocimiento y su impacto en la capacidad de absorción de pymes de baja tecnología. Cuadernos de Administración, 30(55), 7-35. http://dx.doi.org/10.11144/Javeriana.cao30-55.bcica.

* * Doctor en Proyectos de Ingeniería e Innovación de la Universidad Politécnica de Valencia, Valencia, España, 2008. Profesor de Tiempo Completo Departamento de Finanzas y Organizaciones en la Universidad del Norte, Colombia.

Correo electrónico: jaiderv@uninorte.com

*** Doctor en Economía de la Universidad de Barcelona, Barcelona, España, 2011. Profesor de Tiempo Completo Departamento de Economía en la Universidad del Norte, Colombia.

Correo electrónico: Ipoloj@uninorte.edu.co

**** Profesional en Negocios Internacionales de la Universidad del Norte, Barranquilla, Colombia, 2015. Consultora de Innovación Diseño de bienes y Servicios en el Centro de Excelencia en Sistemas de Innovación, Colombia.

Correo electrónico: mcotesa@uninorte.com

$* * * *$ Magister en Economía de la Universidad del Norte, Barranquilla, Colombia, 2016. Asistente de Investigación en el Departamento de Economía de la Universidad del Norte, Colombia.

Correo electrónico:jcvega@uninorte.edu.co
} 


\section{La base de conocimiento y su impacto en la capacidad de absorción de pymes de baja tecnología}

\section{RESUMEN}

Este artículo examina los efectos de la base de conocimiento sobre la capacidad de absorción (CA) de pymes de baja tecnología, teniendo en cuenta el efecto mediador de los antecedentes organizacionales. El estudio empírico se basa en el uso de ecuaciones estructurales a partir de los datos derivados de un cuestionario aplicado a 96 empresas de los sectores agroindustrial y logístico del departamento del Atlántico, Colombia. Los resultados muestran que la base de conocimiento no es suficiente para generar impactos significativos en la CA empresarial, requiriendo de antecedentes organizacionales y, en particular, del desarrollo de redes de comunicación internas (capacidades de socialización) que capitalicen las habilidades de los empleados en beneficio de la capacidad de absorción empresarial.

Palabras clave: capacidad de absorción, base de conocimiento, capacidades de socialización, formalización, innovación, I+D.

Clasificación JEL: M10, O32, L29

Firm's Knowledge Base and its impact on absorptive capacity in Low-tech SMEs

\section{A base de conhecimento e seu impacto na capacidade de absorção de PMEs de baixa tecnologia}

\section{ABSTRACT}

This paper examines the effects of the firm's knowledge base on the absorptive capacity (ACAP) in low-tech SMEs, emphasizing on the mediating role of organizational antecedents. For this purpose, an econometrical approach known as structural equation modeling was undertaken based on data collected from a survey applied to 96 firms belonging to agro-industrial and logistics service sectors from the department of Atlántico, Colombia. Results suggest that knowledge base is not sufficient to generate significant impacts on the firm's ACAP, in fact, it requires the mediation of organizational antecedents. In particular, the deployment of internal communication networks (socialization skills) to capitalize employee's capabilities in benefit of the firm's absorptive capacity.

Keywords: Absorptive capacity, knowledge base, socialization capabilities, formalization, Innovation, R\&D JEL Classification: M10, O32, L29

\section{Resumo}

Este artigo analisa os efeitos da base de conhecimento sobre a capacidade de absorção (CA) de PMEs de baixa tecnologia, levando em conta o efeito mediador dos antecedentes organizacionais. Este estudo empírico está baseado no uso de equações estruturais a partir dos dados derivados de um questionário aplicado a 96 empresas dos setores agroindustrial e logístico do estado do Atlântico, Colômbia. Os resultados mostram que a base de conhecimento não é suficiente para gerar impactos significativos na CA empresarial, requerendo de antecedentes organizacionais e, em particular, do desenvolvimento de redes de comunicação internas (capacidades de socialização) que capitalizem as habilidades dos empregados em prol da CA empresarial.

Palavras-chave: capacidade de absorção; base de conhecimento; capacidades de socialização, formalização; inovação; P+D. Classificação JEL: M1O, O32, L29 


\section{Introducción}

La innovación es concebida hoy día como un proceso interactivo que involucra diversos actores e instituciones (Teece, 1996), y en el cual las capacidades y los recursos internos de la empresa se complementan con las fuentes de conocimiento tecnológico disponibles en el entorno (Caloghirou et ál., 2004). La complejidad y rapidez del cambio técnico han hecho que las empresas recurran cada vez más al conocimiento existente fuera de sus fronteras, para desarrollar productos y servicios acordes con las exigencias del mercado. En este contexto, un enfoque que ha cobrado importancia como marco de análisis para explicar la naturaleza y dinámica de los procesos de innovación es el de la capacidad de absorción.

El concepto de capacidad de absorción fue introducido en la literatura por Cohen y Levinthal (1990), quienes lo definieron como: “la habilidad de la empresa para reconocer el valor de una nueva información externa, asimilarla y aplicarla con fines comerciales" (Cohen y Levinthal, 1990, p. 128). Desde su formulación, este concepto ha cobrado relevancia para explicar diversos fenómenos asociados no sólo con los procesos de innovación empresarial, sino también con la efectividad de las alianzas empresariales (Lane y Lubatkin, 1998) y los procesos de transferencia tecnológica entre países (Mowery y 0xley, 1995).

Un aspecto central en la literatura sobre este tema ha sido la definición de variables que permitan medir la capacidad de absorción a nivel organizacional, así como la identificación de los factores que contribuyen a su desarrollo. En este sentido y pese a la complejidad alrededor de su modelación, parece haber un consenso en la literatura en torno a dos rasgos fundamentales: la naturaleza multidimensional del concepto y su dependencia de la trayectoria pasada (Vega-Jurado et ál., 2008). La primera característica implica no sólo la identificación del conocimiento externo relevante sino también la capacidad de la empresa para adquirir, asimilar y explotar dicho conocimiento. Este carácter multidimensional, condujo a que autores como Zahra y George (2002) definieran la capacidad de absorción como un constructo conformado por dos dimensiones: capacidad de absorción potencial (adquisición y asimilación) y capacidad de absorción realizada (transformación y explotación), destacando con ello la dificultad de medir dicha capacidad en función de una única variable.

La segunda característica constituye uno de los atributos más relevantes de la capacidad de absorción y está asociada con el carácter acumulativo de los procesos de aprendizaje. Esta característica hace que la capacidad de absorción sea más eficiente 
en aquellas áreas en las cuales la empresa tiene tanto conocimiento como experiencia acumuladas. Dicho de otra forma, la capacidad de una empresa para adquirir y explotar el conocimiento externo es mayor en aquellos campos en los cuales posee conocimiento o experiencia previa relacionada. Atendiendo a esta característica se ha destacado la base de conocimiento de la organización como uno de los factores determinantes del desarrollo de su capacidad de absorción. Esta base de conocimiento ha sido tradicionalmente analizada empleando medidas relacionadas con los esfuerzos empresariales en actividades de $I+D$, señalando que el desarrollo de dichas actividades fortalecen la trayectoria tecnológica de la empresa en un campo de conocimiento específico y la hacen más receptiva al conocimiento externo relevante (Veugelers, 1997; Coombs y Bierly, 2006, Cassiman y Veugelers, 2006).

Aunque es innegable el papel de la I+D, no sólo como fuente de innovación sino también como actividad clave para el fortalecimiento de la capacidad de absorción empresarial, lo cierto es que en muchos sectores industriales $y$, en particular, en el contexto de las pequeñas y medianas empresas de países emergentes, los esfuerzos empresariales en esta área son muy limitados. Al mismo tiempo, la evidencia indica que este tipo de empresas tienen una mayor necesidad de buscar conocimientos y recursos complementarios en su entorno próximo -debido a la disposición limitada de recursos internos-y suelen recurrir a diversas fuentes externas de información para el desarrollo de sus actividades innovadoras (Vega-Jurado y Schmutzler, 2017). Lo anterior supone que para las pymes el desarrollo de un buen nivel de capacidad de absorción es un aspecto relevante y, al mismo tiempo, deja en evidencia la necesidad de profundizar en el análisis de sus factores determinantes considerando otros aspectos asociados a la base de conocimiento organizacional más allá de la I+D (García-Granero et ál., 2014a).

En línea con lo anterior, no hay que olvidar que los procesos de innovación se desarrollan en el marco de una organización y, por lo tanto, las capacidades individuales escalan al nivel organizacional a través de prácticas que facilitan la diseminación y trasformación del conocimiento (Kim, 1998). Ello implica que la capacidad de absorción de una empresa no es el resultado de la suma de la capacidad de absorción de los individuos que la conforman, sino que más bien su desarrollo se encuentra determinado por una serie de factores de carácter organizacional. Estos factores se han asociado con las denominadas capacidades combinativas (Van den Bosch et ál., 1999) que incluyen aquellas capacidades que regulan los procesos de comunicación, socialización y coordinación al interior de la organización. Aunque existen diversos trabajos que han explorado el efecto de dichas capacidades (Jansen et ál., 2005; Vega-Jurado et ál., 2008), la investigación 
sobre los antecedentes organizacionales de la capacidad de absorción tiene aún vacíos importantes (Flatten et ál., 2015), especialmente en lo relacionado con la conexión existente entre estos factores y la base de conocimiento de la organización.

El objetivo de este artículo es avanzar en el análisis de los determinantes de la capacidad de absorción partiendo de la consideración de los aspectos antes mencionados. Así, se busca contribuir a la literatura sobre capacidad de absorción analizando cómo las capacidades individuales se complementan e integran en el ámbito organizacional para incidir en el desarrollo de la capacidad de absorción de la empresa. Para ello, se analiza de manera conjunta el efecto de la base de conocimiento y de los antecedentes organizacionales, proponiendo que estos últimos median la relación entre el primero y la capacidad de absorción. Esta relación de mediación ha sido escasamente estudiada en la literatura y, constituye, a juicio de los autores, un elemento clave para explicar el proceso a través del cual se consolida la capacidad de absorción de una organización.

La investigación se lleva a cabo en un contexto de pymes de baja intensidad tecnológica, por lo que el análisis de la base de conocimiento se aborda teniendo en cuenta aspectos más genéricos asociados a la formación y la experiencia de los trabajadores, en lugar de los esfuerzos empresariales en I+D. Al hacer esto, el estudio pretende corregir un sesgo existente en la literatura actual que prioriza la I+D como determinante de la capacidad de absorción y deja en un segundo plano el estudio de los mecanismos a través de los cuales las pymes no intensivas en tecnología explotan e integran su base de conocimiento con el conocimiento disponible en el entorno.

A pesar de la importancia que tiene la capacidad de absorción para empresas de todos los tamaños y sectores, los estudios se han centrado principalmente en grandes empresas o empresas pertenecientes a sectores altamente tecnológicos. Por eso, este artículo hace un aporte al analizar la capacidad de absorción en pequeñas y medianas empresas de baja intensidad tecnológica. Para ello se aplicó un instrumento diseñado específicamente para capturar la naturaleza multidimensional de la capacidad de absorción, así como los constructos asociados con la base de conocimiento y los antecedentes organizacionales.

El artículo se estructura de la siguiente forma: en la primera sección se revisa la literatura y se presentan las principales hipótesis del estudio. En la segunda se expone la metodología, los datos, las variables y la técnica de estimación empleada en la investigación. En la tercera se presentan los resultados, y en la cuarta sección se presentan las conclusiones y las propuestas de futuras líneas de investigación. 


\title{
1. Revisión de la literatura y desarrollo de hipótesis
}

\author{
1.1. La base de conocimiento como determinante \\ de la capacidad de absorción
}

El concepto de capacidad de absorción ha sido empleado en la literatura reciente para explicar fenómenos de diversa naturaleza. Su uso ha sido especialmente prolífico a nivel empresarial, donde ha sido empleado como herramienta para analizar los procesos de innovación y estudiar el efecto del aprendizaje organizacional en la creación de ventajas competitivas sostenibles.

Cohen y Levinthal (1990) fueron los primeros en ofrecer una definición del concepto y en desarrollar un marco teórico general sobre sus características en el ámbito empresarial. Uno de los aspectos señalado por estos autores es el carácter acumulativo de la capacidad de absorción y su dependencia con respecto a la base de conocimiento de la organización. Estos autores destacaron que el conocimiento y experiencia que poseen los empleados en un campo determinado facilita a la organización reconocer la importancia de los avances científicos y tecnológicos y su aplicación para el desarrollo de nuevos productos. Lo anterior supone que la capacidad de absorción es dependiente de la trayectoria pasada o de la historia de la organización. Este planteamiento es, en cierta medida, congruente con los postulados de Nelson y Winter (1982), sobre el papel de las rutinas de comportamiento organizacional y las "trayectorias naturales" de las tecnologías en la visualización de las sendas futuras de desarrollo y crecimiento.

Aunque Cohen y Levinthal (1990) tratan la base de conocimiento en un sentido amplio, relacionándola con las habilidades, experiencia y el lenguaje compartido de los trabajadores, lo cierto es que los estudios empíricos han focalizado la atención en la I+D como variable clave de análisis. De esta forma, se ha generado un cuerpo de literatura que analiza la relación existente entre los esfuerzos internos en I+D y el uso y explotación de las fuentes externas de conocimiento. La mayor parte de los estudios han encontrado una relación positiva entre la I+D interna y la adquisición de conocimiento externo, a través de diversos mecanismos, tales como la compra de tecnología, la cooperación con agentes externos o la subcontratación de actividades de I+D (Arora y Gambardella, 1994; Lowe y Taylor; 1998; Veugelers, 1997; Becker y Dietz, 2004).

A pesar de la importancia de la I+D como determinante de la capacidad de absorción, diversos investigadores han llamado la atención sobre las limitaciones que tiene esta 
variable en los modelos de análisis (Vega-Jurado et ál., 2008). En primer lugar, es ampliamente reconocido que las actividades de I+D constituyen una fuente de innovación en sectores con alta intensidad tecnológica, mientras que en sectores tradicionales de la manufactura, por ejemplo, la intensidad de dichas actividades es mucho menor. Lo anterior se amplifica cuando se analiza el caso de industrias ubicadas en contextos como el latinoamericano donde, a diferencia de los países desarrollados, la mayor parte de los investigadores y la financiación de la I+D no se concentra en el sector empresarial, sino en el sector académico o público. En estos casos los esfuerzos internos en I+D pueden no ser el mejor indicador para capturar la naturaleza y diversidad de base de conocimiento de la organización.

En segundo lugar, tal como se ha planteado anteriormente, la base de conocimiento de la organización se configura a partir de diversos factores que van más allá de la I+D e incluye un conjunto de elementos, a menudo tácitos, relacionados con el nivel educativo, las habilidades de los empleados (Walls y Ungson, 1991) y las experiencias internalizadas (Tripsas y Gavetti, 2000), que actúan como reguladores en el momento de valorar el conocimiento relevante disponible en el entorno. Gray (2006), por ejemplo, encontró que las variables asociadas a la edad y la educación de los trabajadores afectan significativamente la adquisición y asimilación del conocimiento externo en las pymes. De forma similar, Romijn y Albaladejo (2002) destacan la importancia del conocimiento especializado y la formación en áreas asociadas con la ingeniería y tecnología como condición necesaria para el aprendizaje tecnológico y el logro de la excelencia innovadora en pymes de alta tecnología.

Adicionalmente, con relación a la formación de los trabajadores, algunos estudios han señalado que las competencias que contribuyen a la capacidad de absorción no están limitados a la educación formal o a las habilidades gerenciales, particularmente cuando se analiza el caso de empresas que operan en sectores no basados en la ciencia. En esta línea, Vinding (2006) sugiere que la variable sectorial incide significativamente en el efecto del nivel educativo sobre la propensión a innovar y se aliena de esta forma con otras investigaciones que señalan que en algunos casos, más que la formación universitaria, la competencia técnica o la experiencia acumulada se convierten en un factor esencial para la explotación del conocimiento (Leiponen, 2005; Freel, 2005). Así, por ejemplo, Souitaris (2002) encuentra que, además de la proporción de empleados graduados universitarios, ingenieros, científicos y gerentes, la experiencia previa en otras compañías representa una variable fuertemente relacionada con la innovación. 
En términos generales, puede decirse que existe un consenso con relación a que cuanto mayor es la base de conocimiento de la organización, medida en términos de educación o experiencia, mayor es su capacidad para identificar, asimilar y transformar el conocimiento externo disponible, incrementando de esta forma la capacidad de absorción de la empresa (Vinding, 2006). Estos elementos son los que le confieren el carácter path depentent a la capacidad de absorción, ya que marcan las rutas de exploración a seguir durante el proceso de adquisición del conocimiento y regulan el desarrollo de las etapas posteriores. Teniendo en cuenta lo anterior se formula la hipótesis 1.

H1: La base de conocimiento de la organización, analizada en términos de formación y experiencia de sus trabajadores, está positivamente relacionada con la capacidad de absorción de la misma.

\subsection{Los antecedentes organizacionales de la capacidad de absorción}

Además de los elementos señalados anteriormente, otro aporte importante en la conceptualización desarrollada por Cohen y Levinthal (1990) fue la distinción entre la capacidad de absorción individual y la capacidad de absorción empresarial, destacando con ello el papel que cumplen ciertos mecanismos que propician la comunicación y la relación entre los miembros de la empresa. Según Cohen y Levinthal (1990), la capacidad de absorción es un proceso organizacional que depende no sólo de la capacidad de sus individuos sino también de la facilidad con la que fluye y se transfiere el conocimiento entre las diferentes unidades u áreas estratégicas de una organización. Así, la capacidad de absorción es altamente dependiente de las prácticas organizacionales que la empresa adopte, bien sea para facilitar la identificación del conocimiento externo relevante o para promover los procesos de intercambio de conocimiento al interior de la empresa (Flor y Oltra, 2013).

Partiendo de la consideración anterior, Van den Bosch et ál. (1999) proponen uno de los primeros modelos de análisis sobre los determinantes de la capacidad de absorción en el que destacan de forma específica la influencia de lo que ellos denominan "antecedentes organizacionales" de la capacidad de absorción. Estos autores, reconocen que el conocimiento previo es un factor fundamental para el desarrollo de la capacidad de absorción, pero establecen al mismo tiempo que existe una serie de mecanismos organizacionales relacionados con la estructura de la firma (p. ej. matricial, funcional) y con las "capacidades combinativas" que inciden de manera relevante en el proceso. Estas capacidades se refieren a la forma en que las empresas sintetizan y aplican el conoci- 
miento adquirido. Van den Bosch et ál. (1999) identificaron tres tipos de capacidades combinativas: las capacidades de sistemas, las capacidades de coordinación y las capacidades de socialización. Las primeras hacen referencia a los manuales y procedimientos usados para integrar el conocimiento explícito. Las segundas contemplan las relaciones existentes entre los miembros de la organización en los diferentes niveles o áreas y, por último, las capacidades de socialización se refieren a la ideología compartida así como a la interpretación colectiva de la realidad. Estas dos últimas capacidades hacen posible la externalización del conocimiento tácito, mientras que las capacidades de sistemas facilitan la combinación del conocimiento explícito y su distribución al interior de la organización.

La importancia de los factores organizaciones también es reconocida por Zahra y George (2002) en su artículo seminal. Estos autores, al igual que sus antecesores, señalan que la experiencia acumulada es clave para la adquisición y asimilación del conocimiento externo, pero sugieren adicionalmente que los procesos de transformación y explotación de dicho conocimiento dependen de los mecanismos de integración social, los cuales son definidos como el conjunto de prácticas que disminuyen las barreras para el intercambio de información al interior de la organización. Estos mecanismos son similares a las capacidades de socialización propuestas por Van den Bosch et ál. (1999) y contribuyen al libre flujo de información y a la transformación del conocimiento, e incrementan el factor de eficiencia representado por la razón entre las capacidades realizadas (RACAP: transformación y explotación) y las capacidades potenciales (PACAP: adquisición y asimilación).

Partiendo de los dos trabajos antes mencionados, Jansen et ál. (2005) realizan un estudio empírico sobre el efecto de los antecedentes organizacionales sobre la PACAP y RACAP. Estos autores definen una escala de medida para evaluar las diferentes dimensiones de la capacidad de absorción, así como una serie de factores para operacionalizar las capacidades combinativas, y demuestran que los antecedentes organizacionales importan para el desarrollo de la capacidad de absorción, pero que su efecto puede variar en función de la dimensión específica que se considere (PACAP o RACAP).

En un estudio posterior, Vega-Jurado et ál. (2008) proponen un modelo en el que los antecedentes organizacionales son reconocidos como determinantes clave de la capacidad de absorción. En concreto, estos autores identifican la formalización y los mecanismos de integración social, como los parámetros organizativos que inciden en los procesos de transmisión de conocimiento entre los individuos y entre las distintas áreas funcionales 
de la empresa. Estos factores, si bien no son los únicos, ofrecen conjuntamente una representación adecuada de la dimensión relacional de la empresa, y capturan aspectos básicos relacionados con las capacidades combinativas destacadas en trabajos previos. De esta manera, los mecanismos de integración social lo asocian con las capacidades de socialización, mientras que la formalización la definen como uno de los parámetros básicos para evaluar las capacidades de sistemas.

En términos generales, la literatura ha destacado que las capacidades de socialización constituyen un determinante clave para el desarrollo de la capacidad de absorción de la empresa en la medida en que fomentan la comunicación y circulación del conocimiento al interior de la organización. Una empresa en donde se promueve la comunicación efectiva en todos los niveles jerárquicos y se facilita el relacionamiento entre los empleados se encuentra, en principio, en una mejor posición no sólo para identificar posibles fuentes de información externa, sino también para facilitar los procesos de transformación y explotación del conocimiento adquirido.

La formalización, por su parte, puede ser entendida como el grado en el cual los procedimientos, reglas, e instrucciones gobiernan el desarrollo de los procesos organizacionales, o dicho de otra forma, el grado con el cual los comportamientos son programados en función de reglas explícitas (Vega-Jurado et ál., 2008). Una de las principales ventajas de la formalización es que facilita el desarrollo de una memoria organizacional que permite actuar de una manera más ágil en situaciones rutinarias y promueve la codificación e integración del conocimiento a través de procesos estandarizados (García-Granero et ál., 2014). En este sentido, un alto grado de formalización puede incentivar el desarrollo de la capacidad de absorción empresarial, al facilitar la identificación y adquisición de conocimiento externo, gracias a la definición de procesos claros relacionados con la identificación de las necesidades y la selección y adquisición de conocimiento.

En términos generales, la literatura ha señalado ampliamente la importancia que tiene la dimensión organizacional como determinante de la capacidad de absorción empresarial. El desarrollo de la capacidad de absorción requiere la configuración de un entorno organizacional que estimule los procesos de búsqueda, asimilación e intercambio de conocimiento. Dicho entorno se construye a partir de la adopción de prácticas que faciliten el flujo de información entre los individuos (capacidad de socialización) o la definición y seguimiento de procedimientos que guíen el desarrollo de las actividades de gestión y/o producción (formalización/capacidades de sistema). Estas relaciones han sido escasamente contrastadas a través de estudios empíricos rigurosos y, la mayor 
parte de los trabajos realizados hasta la fecha, se han quedado en el debate teórico o han suministrado evidencia preliminar. De acuerdo con esto se plantea la hipótesis 2.

H2: Los antecedentes organizacionales están positivamente relacionados con la capacidad de absorción de la empresa. En particular:

H2a: La capacidad de socialización está positivamente relacionada con la capacidad de absorción de la empresa.

H2b: La formalización está positivamente relacionada con la capacidad de absorción de la empresa.

\subsection{El rol mediador de los antecedentes organizacionales}

Las hipótesis señaladas en los apartados anteriores siguen la línea tradicional de los estudios realizados hasta la fecha en lo que se refiere a los determinantes de la capacidad de absorción empresarial, destacando la relevancia que tiene tanto la base de conocimiento como los antecedentes organizacionales. El efecto directo de estos factores como determinantes de la capacidad de absorción, aunque importante, no es el único que se debe analizar. Partiendo de la consideración que la capacidad de absorción es un proceso que se nutre de las capacidades de los individuos que conforman la empresa pero que finalmente se configura en el ámbito organizacional, proponemos que el efecto de la base de conocimiento debe estudiarse teniendo en cuenta el desarrollo de ciertas capacidades organizacionales. En este sentido, proponemos que, más que un efecto directo, la ruta principal a través de la cual la base de conocimiento influye sobre la capacidad de absorción es gracias al desarrollo de un contexto organizacional que favorece la coordinación y la socialización del conocimiento al interior de la empresa.

El nivel de formación de los trabajadores y su experiencia en un área determinada, hace más fácil la sistematización de los procesos y la definición y adopción de protocolos de actuación, creando así capacidades de sistemas en la organización (formalización). De igual forma, la experiencia y antigüedad de los trabajadores en una empresa favorece la creación de una visión y un sistema de valores compartidos que, a su vez, estimulen el dialogo y la comunicación efectiva en todos los niveles organizacionales (capacidades de socialización) (Van den Bosch et ál., 1999). Por tanto, la base de conocimiento, puede estimular el desarrollo de las capacidades de socialización y de las capacidades de sistemas de una empresa (formalización) y, dado que estas tienen un impacto sobre 
la capacidad de absorción, terminar incidiendo sobre la misma. Es precisamente en este sentido, que proponemos que el efecto de la base de conocimiento sobre la capacidad de absorción se encuentra mediado por los antecedentes organizaciones, en particular, por las capacidades de socialización y el grado de formalización de los procesos.

Este efecto mediador de los antecedentes organizacionales no ha sido analizado en estudios previos y constituye un vacío que el presente artículo pretende subsanar. Múltiples investigadores han llamado la atención sobre la necesidad de examinar más en detalle el efecto de los antecedentes organizacionales (Burcharth et ál., 2015; Flatten et ál., 2015; Volberda et ál., 2010), pero los esfuerzos realizados hasta la fecha se han concentrado en el mejor de los casos, en el análisis del efecto directo de dichos factores. La consideración de su efecto mediador, no sólo permitirá avanzar en este sentido, sino que además permitirá comprender mejor el efecto de la base de conocimiento sobre la capacidad de absorción. En resumen, se plantean la hipótesis 3.

H3: Los antecedentes organizacionales median la relación entre la base de conocimiento y la capacidad de absorción de la empresa.

H3a: Las capacidades de socialización median la relación entre la base de conocimiento y la capacidad de absorción de la empresa.

H3b: El grado de formalización media la relación entre la base de conocimiento y la capacidad de absorción de la empresa.

\section{Metodología}

\subsection{Datos}

El estudio empírico se basa en una muestra de empresas pertenecientes a los sectores logístico y agroindustrial del departamento del Atlántico, Colombia. Se trata de empresas pequeñas y medianas de baja intensidad tecnológica y que dedican pocos esfuerzos al desarrollo de actividades internas de I+D. El estudio de campo fue realizado entre enero y junio de 2016. La encuesta fue diligenciada por el Director del departamento técnico/producción en lo que respecta a las preguntas relacionadas con los esfuerzos en innovación y la capacidad de absorción; y por el director general o gerente en lo que respecta a las cuestiones asociadas con las prácticas organizacionales y estilos de dirección. En total se logró recopilar la información de 96 empresas. 
El cuestionario que se aplicó fue diseñado específicamente para capturar el carácter multidimensional de la capacidad de absorción y analizar las relaciones propuestas en el presente documento. En este sentido, un valor del presente estudio es que las variables que se emplean para analizar las relaciones entre los constructos objeto de esta investigación han sido construidas a partir del uso de preguntas y escalas de medida diseñadas específicamente para tal fin. El instrumento se definió a partir de la revisión de la literatura y contempla escalas validadas en estudios previos (Flatten et ál., 2011; Jansen et ál., 2005; Jimenez-Barrionuevo et ál., 2011).

\subsection{Medidas}

\subsubsection{BASE DE CONOCIMIENTO}

La mayoría de estudios relacionados con la capacidad de absorción ha analizado la base de conocimiento de la organización en términos de los esfuerzos empresariales en el desarrollo de actividades internas de I+D. Como se comentó antes, esta variable tiene muchas limitaciones, en particular cuando se trata de sectores poco intensivos en tecnología y en contextos de economías emergentes. Por esta razón, en este artículo se adopta una perspectiva más amplia y se analiza la base de conocimiento en función de aspectos más homogéneos presentes en cualquier industria, asociados con la cualificación y experiencia de los empleados. De esta forma, se plantea la base de conocimiento como un constructo formado por dos dimensiones (experiencia y formación), cada una definida a partir de un grupo de diferentes ítems evaluados en una escala likert (1: nada de acuerdo, 7: totalmente de acuerdo).

\subsubsection{ANTECEDENTES organizaCionales}

El análisis de los antecedentes organizacionales es realizado teniendo en cuenta dos tipos de capacidades combinativas: las capacidades de socialización y las capacidades de sistemas (Van den Bosch et ál. 1999). Las capacidades de socialización son evaluadas a través de una serie de ítems que capturan el grado en el cual los trabajadores de la compañía se relacionan entre sí y la comunicación fluye entre los diferentes niveles organizativos. Estos ítems se definieron teniendo en cuenta escalas contrastadas en estudios previos (Jansen et ál., 2005). Por su parte, las capacidades de sistemas se analizan a través del grado de formalización de la compañía. Para ello se define una serie de ítems que capturan la existencia de procedimientos formalizados para el desarrollo de las actividades de gestión y producción, así como el cumplimiento sistemático de 
los mismos (Jansen et ál., 2005; García-Granero et ál., 2014b). Cada uno de los ítems incluidos para evaluar los constructos de formalización y capacidades de socialización, son evaluados en una escala Likert (1: nada de acuerdo, 7: totalmente de acuerdo).

\subsubsection{CAPACIDAD DE ABSORCIÓN}

Para medir la capacidad de absorción se parte de la consideración del carácter multidimensional del constructo. En este sentido, en el cuestionario se han planteado diferentes ítems que intentan capturar las cuatro dimensiones básicas de la capacidad de absorción: adquisición, asimilación, trasformación y explotación del conocimiento externo. El diseño de estas preguntas corresponde a una versión adaptada del instrumento de medida desarrollado e implementado en algunos trabajos previos (Flatten et ál., 2011; Jansen et ál., 2005; Jiménez-Barrionuevo et ál., 2011). Todos los ítems son medidos a partir de afirmaciones evaluadas en una escala Likert (1: nada de acuerdo, 7: totalmente de acuerdo) (ver anexo).

\subsection{Técnica de estimación}

Para poder cuantificar el efecto y la importancia que tienen los factores asociados a la base de conocimiento sobre la capacidad de absorción, al igual que el efecto mediador de los antecedentes organizacionales relacionados con los mecanismos de integración socialy el grado de formalización de los procesos, se lleva a cabo una aproximación econométrica conocida como modelación por ecuaciones estructurales. Este tipo de modelos se utilizan a menudo para evaluar constructos latentes no observables, como el que se puede observar en la figura 1, donde el modelo propuesto establece una relación de dependencia entre varias variables (constructos) endógenas y exógenas en el que las relaciones no son lineales sino que existen múltiples interrelaciones entre los elementos que lo configuran.

Mediante un Modelo de Ecuaciones Estructurales se puede combinar el enfoque predictivo de las técnicas clásicas econométricas de regresión múltiple (examinando relaciones de dependencia entre variables) con el enfoque psicométrico, basado en la medición de variables latentes (no observadas) a través de múltiples variables observadas (indicadores), aplicando para ello el análisis factorial. Por lo tanto, en un Modelo de Ecuaciones Estructurales se deben considerar realmente dos tipos de modelo:

- El modelo de medida (que identifica al enfoque psicométrico), es decir, el que resulta de aplicar la técnica de análisis factorial para determinar las cargas fac- 
toriales de las variables observables (indicadores) con relación a las variables latentes (constructos). Estos últimos pueden estar constituidos por indicadores reflectivos o por indicadores formativos. ${ }^{1}$

- El modelo estructural (que identifica las relaciones lineales del enfoque econométrico) permite analizar las relaciones de causalidad entre un conjunto de constructos independientes (exógenos) y dependientes (endógenos).

De esta manera, para poder analizar un modelo de ecuaciones estructurales se debe llevar a cabo un estudio de la validez y fiabilidad del modelo de medida, donde se analice si los conceptos teóricos (representados por los constructos) se estiman correctamente a través de los indicadores de medida, es decir, poder comprobar la validez interna de las mediciones psicométricas para la construcción de variables latentes (constructos); y una valoración del modelo estructural, donde se analizan las relaciones establecidas entre los distintos constructos del modelo teórico.

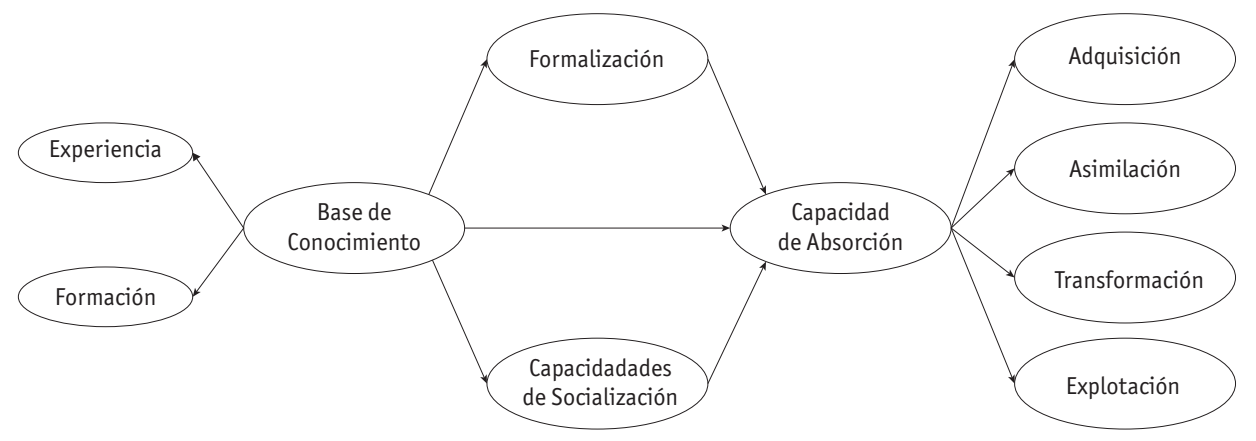

Figura 1. Modelo de Ecuaciones Estructurales

Fuente: elaboración de los autores.

En términos de lo mostrado en la Figura 1, las variables de capacidad de absorción serán medidas a través de modelos estimados de manera simultánea que permitan encontrar los niveles de adquisición, asimilación, transformación y explotación de cada empresa. Dado que no existen valores concretos de estas variables, primero se debe hacer uso de

1 Un indicador formativo se refiere a un índice de una suma ponderada de los valores de las variables. En una construcción formativa, los indicadores causan el constructo, mientras que en una formación reflectiva los indicadores son causados por el constructo o la variable latente. 
constructos teóricos que permitan medir factores relacionados con las cuatro dimensiones. Para los demás constructos se siguen la creación a partir de variables de medición.

\section{Análisis y resultados}

\subsection{Evaluación de las propiedades psicométricas de las escalas de medida}

Respecto a la evaluación del modelo de medida, se debe valorar la fiabilidad individual de los ítem que conforman los constructos de primer orden definidos previamente, examinando su carga factorial ( $\lambda$ ) según lo definido por Hair etál. (2010), que explican que para aceptar un indicador como integrante de un constructo, este ha de poseer una carga mayor a 0,5; es decir, las variables psicométricas con alta correlación con la variable latente.

La tabla 1 muestra los estadísticos descriptivos de las variables incluidas en el modelo, así como los resultados del análisis factorial de las cargas de las variables de medición para el caso de los constructos de primer orden. En términos generales, los resultados indican que los indicadores utilizados para la creación de las variables utilizadas en el modelo estructural son en, su mayoría, fiables. Los ítems asociados con los constructos de formalización, capacidades de socialización y formación muestran un alto grado de fiabilidad. De los constructos analizados, la dimensión de experiencia (asociada con la base de conocimiento) fue la que presentó la menor cantidad de indicadores con carga factorial superior al umbral definido.

Tabla 1

Resultados del Modelo de Medición. Constructos de Primer Orden

\begin{tabular}{|c|c|c|c|c|c|}
\hline \multirow{2}{*}{$\begin{array}{c}\text { Factores } \\
\text { Experiencia }\end{array}$} & Media & Desv. & \multicolumn{2}{|c|}{ Carga Factorial } & Err. Est. \\
\hline & & & & & \\
\hline Bc-Exp-01 & 5,865 & 1,042 & 0,478 & $* * *$ & 0,136 \\
\hline Bc-Exp-02 & 4,479 & 1,508 & 0,271 & ** & 0,179 \\
\hline Bc-Exp-03 & 5,375 & 1,474 & 0,108 & & 0,188 \\
\hline Bc-Exp-04 & 3,438 & 1,770 & $-0,008$ & & 0,176 \\
\hline Bc-Exp-05 & 4,406 & 1,798 & 0,625 & $* * *$ & 0,148 \\
\hline Bc-Exp-06 & 4,792 & 1,507 & 0,758 & $* * *$ & 0,075 \\
\hline \multicolumn{6}{|l|}{ Formación } \\
\hline Bc-For-01 & 5,771 & 1,081 & 0,839 & $* * *$ & 0,078 \\
\hline Bc-For-02 & 5,073 & 1,324 & 0,690 & $* * *$ & 0,142 \\
\hline
\end{tabular}




\begin{tabular}{|c|c|c|c|c|c|}
\hline \multirow{2}{*}{$\begin{array}{c}\text { Factores } \\
\text { Bc-For-03 }\end{array}$} & \multirow{2}{*}{$\begin{array}{l}\text { Media } \\
3,969\end{array}$} & \multirow{2}{*}{$\begin{array}{c}\text { Desv. } \\
2,203\end{array}$} & \multicolumn{2}{|c|}{ Carga Factorial } & \multirow{2}{*}{$\begin{array}{c}\text { Err. Est. } \\
0,137\end{array}$} \\
\hline & & & $-0,200$ & * & \\
\hline Bc-For-04 & 5,271 & 1,525 & 0,558 & $* * *$ & 0,130 \\
\hline Bc-For-05 & 4,885 & 1,806 & 0,619 & $* * *$ & 0,179 \\
\hline \multicolumn{6}{|c|}{ Capacidades de Socialización } \\
\hline CS-01 & 5,094 & 1,459 & 0,764 & $* * *$ & 0,051 \\
\hline CS-02 & 5,854 & 1,124 & 0,700 & $* * *$ & 0,096 \\
\hline CS-03 & 5,917 & 1,270 & 0,840 & ** & 0,076 \\
\hline CS-04 & 5,771 & 1,566 & 0,715 & $* * *$ & 0,107 \\
\hline \multicolumn{6}{|c|}{ Formalización } \\
\hline For-01 & 4,792 & 2,062 & 0,665 & $* * *$ & 0,069 \\
\hline For-02 & 5,104 & 1,593 & 0,759 & $* * *$ & 0,097 \\
\hline For-03 & 4,333 & 2,050 & 0,698 & $* * *$ & 0,121 \\
\hline \multicolumn{6}{|l|}{ Adquisición } \\
\hline Adq-01 & 4,396 & 2,193 & 0,845 & $* * *$ & 0,043 \\
\hline Adq-02 & 3,729 & 2,255 & 0,821 & $* * *$ & 0,045 \\
\hline Adq-03 & 4,323 & 2,060 & 0,864 & $* * *$ & 0,017 \\
\hline Adq-04 & 4,833 & 1,982 & 0,806 & $* * *$ & 0,036 \\
\hline Adq-05 & 3,354 & 2,335 & 0,157 & & 0,371 \\
\hline \multicolumn{6}{|l|}{ Asimilación } \\
\hline Asi-01 & 4,854 & 1,795 & 0,841 & $* * *$ & 0,023 \\
\hline Asi-02 & 4,740 & 1,865 & 0,853 & $* * *$ & 0,018 \\
\hline Asi-03 & 4,552 & 1,776 & 0,84 & $* * *$ & 0,022 \\
\hline Asi-04 & 5,083 & 1,781 & 0,806 & $* * *$ & 0,034 \\
\hline \multicolumn{6}{|c|}{ Transformación } \\
\hline Tra-01 & 4,542 & 1,765 & 0,841 & $* * *$ & 0,019 \\
\hline Tra-02 & 4,615 & 1,767 & 0,846 & $* * *$ & 0,017 \\
\hline Tra-03 & 5,198 & 1,505 & 0,779 & $* * *$ & 0,035 \\
\hline Tra-04 & 4,875 & 1,700 & 0,823 & $* * *$ & 0,024 \\
\hline \multicolumn{6}{|l|}{ Explotación } \\
\hline Exp-01 & 3,885 & 1,886 & 0,837 & $* * *$ & 0,023 \\
\hline Exp-02 & 4,396 & 1,911 & 0,82 & $* * *$ & 0,028 \\
\hline Exp-03 & 3,615 & 1,991 & 0,819 & $* * *$ & 0,044 \\
\hline Exp-04 & 2,760 & 1,851 & $-0,649$ & $* * *$ & 0,132 \\
\hline
\end{tabular}

Significancia $99 \% * * *, 95 \% * *, 90 \% *$

Fuente: elaboración propia. 
Para el caso de los constructos de segundo orden (capacidad de absorción y base de conocimiento), sólo se verifica la significancia de la relación que estos tienen con los de primer orden. En este caso, los ítems empleados para medir las diferentes dimensiones de la capacidad de absorción (adquisición, asimilación, transformación y explotación) muestran un alto grado de fiabilidad, al tiempo que dichas dimensiones muestran ser adecuadas para la construcción del constructo general de capacidad de absorción. Lo mismo ocurre con los constructos de formación y experiencia utilizados para capturar de manera conjunta la base de conocimiento de la empresa (tabla 2).

\section{Tabla 2}

Resultados del Modelo de Medición. Constructos de Segundo Orden

\begin{tabular}{|c|c|c|c|}
\hline Constructos & \multicolumn{2}{|c|}{ Coeficiente } & Err. Est. \\
\hline \multicolumn{4}{|c|}{ Capacidad de Absorción } \\
\hline Adquisición & 0,803 & $* *$ & 0,067 \\
\hline Asimilación & 0,828 & * & 0,395 \\
\hline Transformación & 1,586 & $* * *$ & 0,507 \\
\hline Explotación & 0,583 & $* *$ & 0,286 \\
\hline \multicolumn{4}{|c|}{ Base de Conocimiento } \\
\hline Formación & 0,533 & $* * *$ & 0,180 \\
\hline Experiencia & 0,521 & $* * *$ & 0,060 \\
\hline
\end{tabular}

Significancia $99 \% * * *, 95 \% * *, 90 \% *$

Fuente: elaboración propia.

En general, los resultados del modelo de medición revelan que los constructos empleados en el modelo de investigación tienen un alto grado de fiabilidad y corroboran la validez del instrumento diseñado para capturar dichos factores en las empresas analizadas.

\subsection{Evaluación del modelo estructural}

Las estimaciones derivadas del modelo estructural, presentadas en la figura 2 y tabla 3 , permiten evaluar los efectos directos e indirectos de la base de conocimiento sobre la capacidad de absorción.

En primer lugar, se evidencia que el constructo base de conocimiento, conformado por las variables formación y experiencia, no se encuentra positivamente relacionado con la capacidad de absorción, al menos cuando se considera su efecto directo. Este resultado, en principio, no respalda la hipótesis 1. 
En lo que respecta a la relación entre los antecedentes organizacionales y la capacidad de absorción de la empresa, los resultados ofrecen evidencia parcialmente a favor de la hipótesis 2. Por una parte, las capacidades de socialización presentan un efecto significativo y positivo sobre la capacidad de absorción; mientras que el coeficiente para formalización no es significativo.

Por otra parte, los resultados ponen de manifiesto que la base de conocimiento se encuentra relacionada con los antecedentes organizacionales. Tanto para formalización como para las capacidades de socialización, la relación con la base de conocimiento es significativa y positiva.

Finalmente, en lo que respecta a la hipótesis 3 , los resultados ofrecen evidencia parcialmente a favor. En concreto, los resultados muestran que las capacidades de socialización median la relación entre la base de conocimiento y la capacidad de absorción, mientras que formalización no ejerce ningún efecto mediador. De hecho, al considerar el efecto mediador de las capacidades de socialización, se encuentra que la base de conocimiento tiene un efecto (indirecto) significativo y positivo sobre la capacidad de absorción.

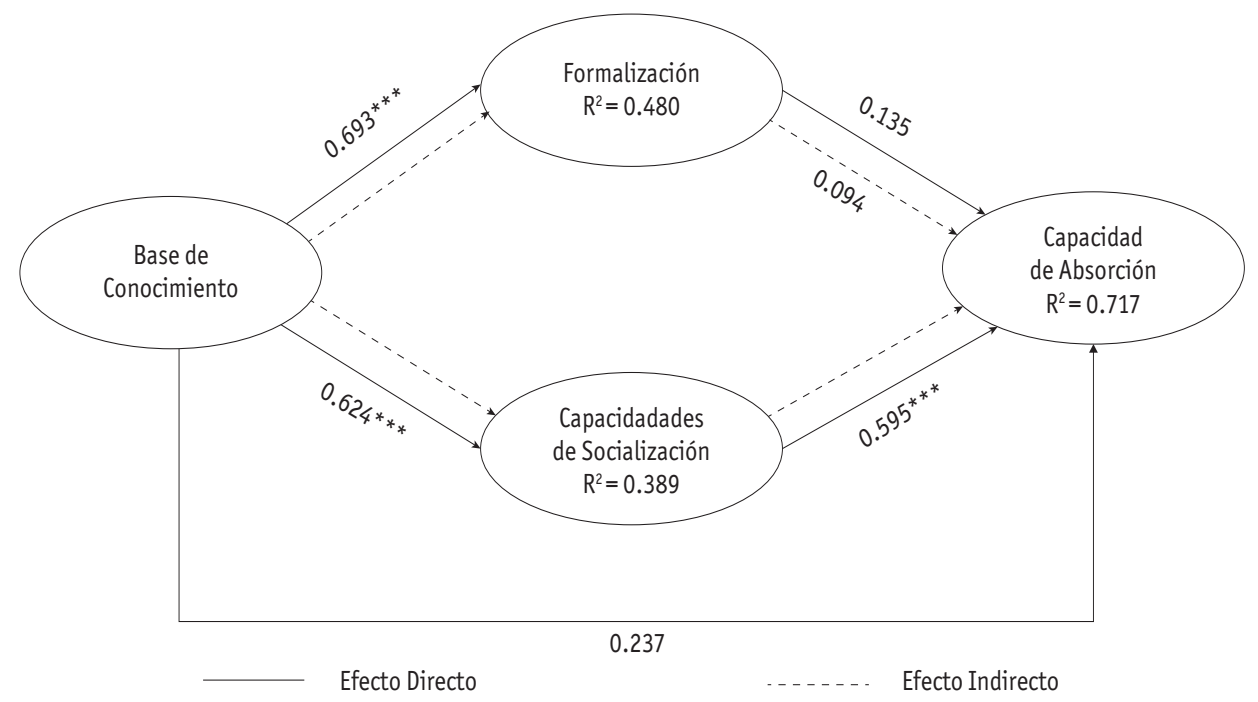

Significa $99 \% * * *, 95 \% * *, 90 \% *$

\section{Figura 2. Resultados del Modelo de Ecuaciones Estructurales}

Fuente: elaboración propia. 


\section{Tabla 3}

Resultados del Modelo de Estructural

\begin{tabular}{|c|c|c|c|c|}
\hline \multicolumn{3}{|r|}{ Trayectoria } & \multicolumn{2}{|c|}{ Coeficiente } \\
\hline \multicolumn{5}{|l|}{ Efectos Directos } \\
\hline \multirow{3}{*}{ Base de Conocimiento } & \multirow{3}{*}{$-->$} & Capacidad de Absorción & 0,237 & \\
\hline & & Capacidades de socialización & 0,624 & *** \\
\hline & & Formalización & 0,693 & *** \\
\hline $\begin{array}{l}\text { Mecanismos de } \\
\text { Integración Social }\end{array}$ & $-->$ & Capacidad de Absorción & 0,595 & *** \\
\hline Formalización & $-->$ & Capacidad de Absorción & 0,135 & \\
\hline \multicolumn{5}{|l|}{ Efectos Indirectos } \\
\hline \multirow{2}{*}{ Base de Conocimiento } & & Capacidades de Socialización --> Capacidad de Absorción & 0,371 & *** \\
\hline & & Formalización --> Capacidad de Absorción & 0,094 & \\
\hline
\end{tabular}

Significancia $99 \% * * *, 95 \% * *, 90 \% *$

Fuente: elaboración propia.

\section{Discusión y conclusiones}

Este artículo responde a uno de los vacíos identificados en la literatura sobre los determinantes de la capacidad de absorción empresarial en pymes de baja intensidad tecnológica. El principal aspecto novedoso del estudio es que no sólo considera el efecto directo de la base de conocimiento y los antecedentes organizacionales, sino que además aborda el posible efecto mediador que ejercen los antecedentes organizacionales sobre la relación entre la base de conocimiento y la capacidad de absorción.

En comparación con los estudios previos, este trabajo se aparta de la tradición de analizar la base de conocimiento en términos de los esfuerzos empresariales en actividades de I+D, al considerar tal como lo han puesto de manifiesto diversos autores, que esta es una medida muy restrictiva, que puede ser poco relevante en el caso de empresas pertenecientes a sectores industriales poco intensivos en tecnología. En su lugar, la base de conocimiento es analizada considerando la formación y la experiencia de los trabajadores, aspectos que incluso fueron inicialmente señalados por Cohen y Levinthal (1990) en su trabajo seminal como elementos clave de la misma.

Contrario a lo planteado en la literatura, los resultados obtenidos muestran que la base de conocimiento no tiene un efecto directo sobre la capacidad de absorción empresarial. Pero, en línea con la hipótesis central de esta investigación, los resultados ofrecen 
evidencia a favor de la existencia de un efecto indirecto de esta variable, mediada por los antecedentes organizacionales y en concreto por las capacidades de socialización. Dicho de otra forma, la base de conocimiento influye sobre la capacidad de absorción en la medida en que estimula el desarrollo de capacidades que promueven la difusión y socialización del conocimiento en la organización. Un corolario de este resultado es que no basta con tener individuos altamente cualificados o con una amplia experiencia en la organización para estimular los procesos de absorción de conocimiento externo. Se requiere el desarrollo de un contexto organizacional que promueva el flujo de conocimiento entre dichos individuos de cara a sacar provecho del conocimiento disponible externamente. Si bien esta relación de mediación no había sido analizada en trabajos previos, los resultados obtenidos están en línea con lo que Van den Bosch et ál. (1999) plantearon con relación a la complementariedad existente entre el conocimiento previo y las capacidades combinativas.

De manera específica, los resultados muestran que la base de conocimiento tiene una relación positiva con la formalización y con las capacidades de socialización, las cuales son los dos antecedentes organizacionales considerados en el estudio. Al mismo tiempo, las capacidades de socialización se encuentran positivamente relacionados con la capacidad de absorción, resultado que está en línea con la hipótesis planteada y con lo sugerido en trabajos previos (Jansen et ál., 2005; Vega-Jurado, 2008; Zahra y George, 2002).

No obstante, a diferencia de lo planteado, el grado de formalización de los procesos, no resultó ser significativo para el desarrollo de la capacidad de absorción de la organización. Esto se podría deber a que un alto grado de formalización puede derivar en estructuras más rígidas que actúen como obstáculos para los procesos de transformación y explotación del conocimiento externo, los cuales constituyen dos dimensiones de la capacidad de absorción. La forma como se midió la capacidad de absorción en esta investigación fue a partir de la consideración del constructo en sus cuatro dimensiones. En este sentido, puede ser posible que la formalización tenga un efecto positivo sobre adquisición y asimilación y un efecto negativo sobre las otras dos dimensiones (transformación y explotación) y, como resultado, el efecto total sobre la capacidad de absorción sea no significativo. Esta explicación es especulativa y requiere, por supuesto, de una investigación más detallada.

En general, el resultado más importante del estudio está asociado con el efecto mediador de los antecedentes organizacionales, lo cual responde a una solicitud expuesta en la literatura reciente con relación a la necesidad de investigaciones que profundicen en el 
análisis de la forma como las capacidades individuales trascienden en el contexto organizacional para estimular la capacidad de absorción de una empresa (Burchartd et ál., 2014; 0jo et ál., 2017; Tortoriello, 2015; Volverda et ál., 2010). Una de las principales implicaciones de dicho resultado es que el desarrollo de la capacidad de absorción es un proceso de carácter organizacional que si bien se nutre de la capacidad de los individuos, depende ante todo de aspectos asociados con el contexto organizacional en el que se desenvuelven los trabajadores. De esta forma, aunque se cuente con personal calificado en términos de educación y experiencia, la carencia de espacios y estructuras que fomenten el intercambio y facilite el flujo de información restringe la capacidad de absorber nuevo conocimiento externo. En este sentido, en caso de no existir un contexto organizacional adecuado, se podría llegar a una situación en la que las capacidades individuales de los trabajadores no logran escalar a la organización por la falta de mecanismos que conduzcan a su explotación.

Los resultados obtenidos tienen implicaciones importantes desde el punto de vista práctico: en un contexto de baja intensidad tecnológica con sectores industriales poco intensivos en $I+D$, las empresas, particularmente pymes, tienen una mayor necesidad para acceder y explotar el conocimiento disponible en su entorno. Para desarrollar dicha capacidad, las empresas deben propender por mejorar el grado de formación y cualificación de sus trabajadores, pero al mismo tiempo deben fomentar el desarrollo de espacios y prácticas de gestión que estimulen la comunicación al interior de la organización. De esta forma, los modelos tradicionales de gestión que normalmente giran en torno a la formación y habilidades funcionales de los empleados, deben expandirse para dar paso a la generación de redes de colaboración que logren externalizar el conocimiento tácito, así como el diseño de sistemas que faciliten el acceso y el flujo del conocimiento explícito.

Lo anterior cobra mayor relevancia en un contexto donde las pymes representan el grueso de las unidades económicas y, como en el caso analizado, un gran porcentaje de las mismas son empresas de propiedad familiar por lo que la distancia de poder puede ser relativamente alta. En este escenario, los gerentes deben preocuparse no sólo por mejorar las capacidades de los trabajadores, sino que también deberían prestar una mayor atención al desarrollo de un contexto organizacional en donde la colaboración y la comunicación se realiza de manera efectiva en todos los niveles de la empresa.

Una de las limitaciones del presente estudio radica en que las medidas relacionadas con los antecedentes organizacionales se encuentran basadas en la percepción del Director o 
Gerente de la compañía. Si bien, estudios previos han empleado metodologías similares, sería interesante complementar estos análisis con información derivada de diferentes encuestados (trabajadores en diferentes niveles jerárquicos). Adicionalmente, los datos empelados son de carácter transversal, lo cual sólo nos permite analizar la situación en un momento específico del tiempo y no realizar análisis de carácter longitudinal.

Además de intentar subsanar las limitaciones metodologías antes señaladas, investigaciones futuras pueden enfocarse en el análisis del efecto de los factores analizados (base de conocimiento, antecedentes organizacionales) sobre las diferentes dimensiones de la capacidad de absorción o, por lo menos, distinguiendo entre capacidad de absorción potencial y capacidad de absorción realizada. De igual forma, resulta también de interés analizar el efecto de los antecedentes organizacionales considerando aspectos relacionados con la dinámica del entorno en el que compite la empresa o la existencia de crisis, todos ellos aspectos que pueden actuar como activadores de los procesos de búsqueda, adquisición y explotación de conocimiento externo.

Agradecimientos. Los autores agradecen el apoyo recibido en el marco del proyecto: “Implementación de un programa de gestión de la innovación empresarial para fortalecer las pymes de sectores estratégicos, Atlántico Caribe (BPIN: 2014000100026; Convenio 0103*2015*000028)", financiado por el Fondo de Ciencia, Tecnología e Innovación del Sistema General de Regalías, Departamento del Atlántico y el apoyo recibido por Colciencias en el marco del proyecto: “Dinámicas de Innovación en Contextos Poco Intensivos en I+D: el caso de la Industria Manufacturera Colombiana (Cod: 121577657885)".

\section{Referencias}

Arora, A. \& Gambardella, A. (1994). Evaluating technological information and utilizing it: Scientific knowledge, technological capability and external linkages in biotechnology. Journal of Economic Behavior and Organization, 24, 91-14. https://doi.org/10.1016/01672681(94)90055-8

Becker, W. \& Dietz, J. (2004). R\&D cooperation and innovation activities of firms-evidence for the German industry. Research Policy, 33, 209-223. https://doi.org/10.1016/j.respol.2003.07.003

Burcharth, A.L.L.D.A., Lettl, C. \& Ulhøi, J.P. (2015). Extending organizational antecedents of absorptive capacity: Organizational characteristics that encourage experimentation. 
Technological Forecasting and Social Change, 90, 269-284. https://doi.org/10.1016/j. techfore.2013.12.024

Caloghirou,Y., Kastelli, I. \& Tsakanikas, A. (2004). Internal capabilities and external knowledge sources: Complements or substitutes for innovative performance? Technovation, 24(1), 29-39. https://doi.org/10.1016/S0166-4972(02)00051-2

Cassiman, B. \& Veugelers, R. (2006). In search of complementarity in innovation strategy: Internal R\&D and external knowledge acquisition. Management Science, 52, 68-82. https:// doi.org/10.1287/mnsc.1050.0470

Cohen, W. M. \& Levinthal, D. A. (1990). Absorptive capacity: A new perspective on learning and innovation. Administrative Science Quarterly, 35(1), 128-152. https://doi. org/10.2307/2393553

Cohen,W. M. \& Levinthal, D. A. (1989). Innovation and Learning: The two faces of R\&D. The Economic Journal, 99, 569-596. https://doi.org/10.2307/2233763

Coombs, J. E. \& Bierly, P. E. (2006). Measuring technological capability and performance. $R \& D$ Maanagment, 36(4), 421-438. https://doi.org/10.1111/j.1467-9310.2006.00444.x

Flatten, T., Adams, D. \& Brettel, M. (2015). Fostering absorptive capacity through leadership: A cross-cultural analysis. Journal of World Business, 50, 519-534. https://doi.org/10.1016/j.jwb.2014.08.010

Flatten, T., Engelen., A., Zahra, S. \& Brettel, M. (2011). A measure of absorptive capacity: Scale development and validation. European Management Journal, 29, 98-116. https://doi. org/ 10.1016/j.emj.2010.11.002

Flor, M. L. \& Oltra., M. (2013). An exploratory analysis of the relationship between absorptive capacity and business strategy. Technology Analysis \& Strategic Management, 25(9), 1103-1117. http://dx.doi.org/10.1080/09537325.2013.832743

Freel, M. S. (2005). Patterns of innovation and skills in small firms. Technovation, 25(2), 123-134.

García-Granero, A., Vega-Jurado, J. \& Alegre-Vidal, J. (2014a). Is R\&D enough to take advantage from external knowledge? Focusing on coordination Mechanisms. Journal of Technology management and Innovation, 9(2), 118-130. https://doi.org/ 10.4067/S071827242014000200009

García-Granero, A., Vega-Jurado, J. \& Alegre-Vidal, J. (2014b). Shaping the firm 's external search strategy. Innovation, 16(3), 417-429. https://doi.org/10.1080/14479338.20 14.11081997

Gray, C. (2006). Absorptive capacity, knowledge management and innovation in entrepreneurial small firms. International Journal of Entrepreneurial Behavior \& Research, 12(6), 345360. https://doi.org/10.1108/13552550610710144

Hair, J. F., Black, W. C., Babin, B. J. \& Anderson, R. E. (2010). Multivariate data analysis (7 ed.). Upper Saddle River, NJ, USA: Prentice-Hall, Inc. 
Jansen, J., Van den Bosch., F. \& Volverda., H. (2005). Managing potential and realized absorptive capacity; how do organizational antecedents matter? Academy of Management Journal, 48(9), 999-1015. http://dx.doi.org/10.5465/amj.2005.19573106

Jiménez-Barrionuevo, M., García-Morales, V. \& Molina, L. (2011). Validation of an instrument to measure absorptive capacity. Technovation, 31, 190-202. http://dx.doi.org/10.1016/j. technovation.2010.12.002

Kim, L. (1998). Crisis construction and organizational learning: Capability building in catching-up at Hyundai Motor. Organization Science, 9(4), 506-521. http://dx.doi.org/10.1287/ orsc.9.4.506

Lane, P. J. \& Lubatkin, M. (1998). Relative absorptive capacity and inter-organizational learning. Strategic Management Journal, 19, 461-477. https://doi.org/10.1002/(SICI)109 7-0266(199805)19:5<461::AID-SMJ953>3.0.C0;2-L

Leiponen, A. (2005). Skills and innovation. International Journal of Industrial Organization, 23(5), 303-323. http://dx.doi.org/10.1016/j.ijindorg.2005.03.005

Lowe, J. \& Taylor, P. (1998). R\&D and technology purchase through licence agreements: complementary strategies and complementary assets. R\&D Management, 28(4), 263-278. http://dx.doi.org/10.1111/1467-9310.00103

Mowery, D. C. \& Oxley, J. E. (1995). Inward technology transfer and competitiveness: The role of national innovation systems. Cambridge Journal of Economics, 19, 67-93. https:// doi.org/10.1093/oxfordjournals.cje.a035310

Nelson, R. R. \& Winter, S. G. (1982). An Evolutionary Theory of Economic Change. Harvard University Press, Cambridge, Mass.

0jo, A. 0., Raman, M. \& Chong, C. W. (2017). Microlevel antecedents of absorptive capacity in joint projects engineering teams. Management Research Review, 40(9), 990-1006. http://dx.doi.org/10.1108/MRR-08-2016-0202

Romijn, H. \& Albaladejo, M. (2002). Determinants of innovation capability in small electronics and software firms in southeast England. Research policy, 31(7), 1053-1067. http://dx. doi.org/10.1016/S0048-7333(01)00176-7

Souitaris, V. (2002). Firmspecific competencies determining technological innovation: A survey in Greece. R\&D Management, 32(1), 61-77. https://doi.org/10.1111/1467-9310.00239

Teece, D. (1996). Firm organization, industrial structure and technological innovation. Journal of Economic Behaviour and Organisation, 31, 193-224. http://dx.doi.org/10.1016/ S0167-2681(96)00895-5

Tortoriello, M. (2015). The social underpinnings of absorptive capacity: the moderating effects of structural holes on innovation generation based on external knowledge. Strategic Management Journal, 36(4), 586-597. http://dx.doi.org/10.1002/smj.2228 
Tripsas, M. \& Gavetti, G. (2000). Capabilities, cognition and inertia: Evidence from digital imaging. Strategic Management Journal, 21, 1147-1162. http://dx.doi.org/10.1002/1097-0266(200010/11)21:10/11\%3C1147::AID-SMJ128\%3E3.0.C0;2-R

Valentim, L., Veríssimo, L. \& Franco, M. (2015). Knowledge management practices and absorptive capacity in small and medium-sized enterprises: is there really a linkage? $R \& D$ management, 46(4), 711-725. https://doi.org/10.1111/radm.12108

Van den Bosch, F. A., Volberda, H. W. \& De Boer, M. (1999). Coevolution of firm absorptive capacity and knowledge environment: Organizational forms and combinative capabilities. Organization Science, 10(5), 551-568. http://dx.doi.org/10.1287/orsc.10.5.551

Vega-Jurado, J. y Schmutzler, J. (2017). Determinantes del uso de fuentes externas de conocimiento en los procesos de innovación empresarial: la importancia de la dimensión regional. Espacios, 38(19), 23-41.

Vega-Jurado, J., Gutiérrez-Gracia, A. \& Fernández-de-Lucio, I. (2008). Analyzing the determinants of firm's absorptive capacity: beyond R\&D. R\&D Management, 38(4), 392-405. https://doi.org/10.1111/j.1467-9310.2008.00525.x

Veugelers, R. (1997). Internal R\&D expenditures and external technology sourcing. Research Policy, 26(3), 303-315. http://dx.doi.org/10.1016/S0048-7333(97)00019-X

Vinding, A. L. (2006). Absorptive capacity and innovative performance: A human capital approach. Economics of Innovation and New Technology, 15(4-5), 507-517. http://dx.doi. org/10.1080/10438590500513057

Volberda, H., Foss, N. \& Lyles, M. (2010). Absorbing the concept of absorptive capacity: How to realize its potential in the organization field. Organization Science, 21(4), 931-951. https://doi.org/10.1287/orsc.1090.0503

Walls ${ }_{\perp}$ J. P. \& Ungson, G. R. (1991). Organizational memory. Academy of Management Review, $16(1), 57-91$.

Zahra, S. A. \& George, G. (2002). Absorptive capacity: A review, reconceptualization, and extension. Academy of Management Review, 27(2), 185-203. http://dx.doi.org/10.5465/ amr.2002.6587995

\section{Anexo. Items incluidos en el cuestionario}

\begin{tabular}{lc}
\hline Items para Base de conocimiento: Experiencia & Codificación \\
\hline $\begin{array}{l}\text { Los empleados de la compañía tienen una amplia experiencia en el de- } \\
\text { sarrollo de las actividades propias de la empresa }\end{array}$ & Bc-Exp-01 \\
$\begin{array}{l}\text { Los empleados de la compañía conocen ampliamente el comportamiento } \\
\text { del mercado }\end{array}$ & Bc-Exp-02
\end{tabular}


Los empleados han adquirido la mayor parte de su experiencia en la compañía

Los empleados han adquirido la mayor parte de su experiencia en otras empresas del sector

La antigüedad media de los empleados de la empresa supera el promedio de las empresas del sector

Comparado con la competencia, nuestros empleados poseen una mayor experiencia

Bc-Exp-06

\section{Items para Base de conocimiento: Formación}

Los empleados de la compañía tienen la formación y el entrenamiento necesario para el desarrollo de sus funciones

Bc-For-01

Comparado con las empresas del sector, nuestros empleados tienen un mayor nivel de formación y entrenamiento

La falta de personal cualificado es una debilidad importante de la empresa

Bc-For-03

La empresa tiene una gran capacidad para retener su personal cualificado

Bc-For-04

La empresa promueve activamente la formación, actualización y entrenamiento de los empleados

Bc-For-05

\section{Items para Capacidades de socialización}

La comunicación es efectiva en todos los niveles de la empresa

En esta empresa los trabajadores tienen plena libertad y se sienten cómodos contactando a otros trabajadores cuando necesitan de su ayuda

En esta empresa es fácil hablar con cualquiera en búsqueda de apoyo independientemente de su posición o cargo

Los directores de un departamento pueden fácilmente organizar reuniones con otros directores para tratar problemas o situaciones de interés para la compañía

\section{Items para Formalización}

Existen procedimientos formalizados para el desarrollo de las actividades de gestión y/o producción

Se siguen de manera sistemática las normas y procedimientos establecidos For-02 
Se realizan evaluaciones continuas a los trabajadores con base en el cumplimiento de las normas y procedimientos establecidos

For-03

\section{Items para Adquisición}

En nuestra empresa se motiva a los empleados a que busquen información de fuentes pertenecientes a la industria

Adq-01

En nuestra empresa se busca regularmente información proveniente de actores especializados (consultores, universidades etc.)

Adq-02

La búsqueda de información externa relevante relacionada con el desempeño del negocio es una actividad cotidiana.

Adq-03

En la empresa no existen mecanismos para la captación del conocimiento externo.

Adq-04

En nuestra empresa se recoge información sobre la industria a través de canales informales (comidas, charlas, etc.)

Adq-05

\section{Items para Asimilación}

En nuestra empresa la información que se obtiene de fuentes externas fluye rápidamente entre los diferentes departamentos.

En nuestra empresa se promueve la difusión y discusión de la información adquirida de fuentes externas entre los diferentes departamentos.

En nuestra empresa se alcanza un entendimiento colectivo de la información y del conocimiento que es adquirido a partir de fuentes externas

El equipo directivo de la empresa promueve la realización de reuniones periódicas entre los departamentos para intercambiar ideas, desarrollos, problemas y logros.

\section{Items para Transformación}

Nuestros empleados tienen la capacidad de estructurar y utilizar la información y el conocimiento adquirido externamente.

Nuestros empleados tienen la capacidad de trasformar el conocimiento adquirido externamente a partir de la base de conocimiento existente en la empresa.

Nuestros empleados son capaces de aplicar el conocimiento adquirido externamente en su trabajo práctico. 
Nuestros empleados tienen la capacidad de generar nuevo conocimiento a partir del conocimiento adquirido externamente.

Tra-04

\section{Items para Explotación}

Nuestra empresa tiene la capacidad de convertir la información y el conocimiento adquirido externamente en innovaciones exitosas.

Nuestra empresa tiene la habilidad de adoptar nuevas tecnologías y desarrollar procesos más eficientes.

En nuestra empresa existen mecanismos que promueven el desarrollo de prototipos de nuevos productos (bienes o servicios)

Nuestra empresa tiene problemas para utilizar la información externa en el desarrollo de nuevos productos (bienes o servicios) o procesos. 